\title{
Energy spectra of electrons and gamma rays produced by the electron accelerator in thunderclouds
}

Ashot Chilingarian ( $\sim$ chili@aragats.am )

Yerevan Physics Institute https://orcid.org/0000-0002-2018-9715

\section{Gagik Hovsepyan}

Yerevan Physics Institute

MAry Zazyan

Yerevan Physics Institute

\section{Article}

Keywords: thuderclouds, electron accelerator, gamma rays, electrons

Posted Date: December 1st, 2020

DOI: https://doi.org/10.21203/rs.3.rs-115135/v1

License: (c) (i) This work is licensed under a Creative Commons Attribution 4.0 International License. Read Full License 


\section{Abstract}

We measure energy spectra of electrons and gamma rays of electromagnetic avalanches developed in the electrified atmosphere as they arrive at the earth's surface at $3200 \mathrm{~m}$ height where Aragats research station is located. We compare intensities and spectra shapes of 2 thunderstorm ground enhancements (TGE) observed in June and September 2020 with simulated ones. Although, the variants of electric field strength and topology assumed in the simulations are too simplified to reproduce the rather complicated and dynamic nature of the atmospheric electric field the closeness of several measured and observed parameters allows us to confirm that the relativistic runaway electron avalanches (RREA) is the origin of TGE, and to outline most probable characteristics of the atmospheric electric field for particular observed TGE events.

\section{Introduction}

In the electric fields in thunderclouds, seed electrons from an ambient population of cosmic rays are accelerated and form electron-gamma ray avalanches, directed either downwards to the Earth's surface or upwards into open space, depending on the direction of the electric field. Intense fluxes of gamma rays observed in space are called Terrestrial Gamma Flashes [TGFs, (Fishman et al. 1994; Briggs et al. 2011; Tavani et al. 2011)], in the atmosphere, they are called gamma glows (McCarthy and Parks 1985; Eack et al. 1996; Kelley et al. 2015; Kochkin et al. 2017), on the ground, they are called Thunderstorm Ground Enhancements [TGEs, (Alexeenko et al. 2002; Chilingarian et al. 2010, 2011; Torii et al. 2011; Tsuchiya et al. 2013; Kuroda et al. 2016; Kudela et al. 2017, Chum et al., 2020, Shepetov et al., 2020). In the latter, also neutron fluxes are observed (Gurevich et al. 2012; Tsuchiya et al. 2012; Chilingarian et al. 2012; Enoto et al. 2017). Very often particle avalanches precede lightning flashes (Chilingarian et al. 2017b). The duration of these fluxes ranges from milliseconds to a few hours and consists of billions of particles (Mailyan et al. 2016; Chilingarian et al. 2017a, 2018). Runaway Breakdown [RB, (Gurevich et al. 1992)], also referred to as Relativistic Runaway Electron Avalanche [RREA, (Babich et al. 2001, 2004; Dwyer 2003)], is the only theoretical model satisfactorily explaining "cloud electron accelerators". However, due to the scarcity of measurements and poor knowledge of the electric structures in the clouds, these phenomena are not well understood until now. On the basis of continuous monitoring of particle fluxes, electric fields, and meteorological conditions on Aragats mountain (Chilingarian et al. 2016) we discover that particle fluxes from the thunderclouds are much larger and extended than expected from the previous observations due to 222Rn progeny circulation in the thunderous atmosphere (Chilingarian et al., 2019, 2020). In this letter, we present precise measurements of the electron and gamma ray energy spectra made by the large scintillation spectrometer along with detailed simulations with CORSIKA code of the RREEA process in the electrified atmosphere. We demonstrate that electromagnetic avalanche simulation with realistic strengths of the electric field yields particle fluxes quite compatible with the measured ones, thus, proving the validity of the RREA origin of the TGE phenomenon.

\section{Energy Spectra Analysis Of Rrea Electrons And Gamma Rays}


Measurement of the energy spectrum of RREA electrons and positrons is a rather difficult problem. After exiting the region of strong electric field where electrons are accelerated and multiplied, the intensity of the electron beam rapidly declines due to ionization losses. On the other hand, gamma rays are attenuated much slower; thus, at the earth's surface, after propagation over few hundreds of meters the intensity of gamma rays is 20-30 times larger than the intensity of electrons (if any).

Thus, Nal spectrometers, having a very small area (usually $0.01-0.03 \mathrm{~m}^{2}$ ) measure usually only gamma ray flux, and, sometimes, where TGE is extremely strong also a small fraction of electrons. Therefore, at present the only spectrometer capable to register RREA electrons and recover their energy spectrum is the ASNT detector located at Mt. Aragats, see details in (Chilingarian et al., 2017). The detector comprises a 4 $\mathrm{m}^{2}$ and $60 \mathrm{~cm}$ thick plastic scintillator (more than 100 times bigger than the largest Nal crystal used in atmospheric high-energy physics measurements) and has the possibility to separate charged and neutral particles. From the ASNT detector, we obtained a 2-second time series of count rate and 20-second time series of histograms of energy releases in a $60-\mathrm{cm}$ thick plastic scintillator. After performing a full GEANT4 simulation of the detector response function we recover differential and integral energy spectra of RREA electrons and gamma rays. Two TGEs observed in Summer-Autumn 2020 were selected for the analysis presented in this study. These TGE events occurred in absolutely different conditions of the atmospheric electric field, one was terminated by the lightning flash, the other declined naturally at LPCR contraction. On 27 June 2020, a large storm lasting 2.5 hours occurred on Aragats. Nearby lightning flashes (nearest to the distance of $\approx 7 \mathrm{~km}$ ) occurred during the first hour of the storm. Attempts to start TGE began at 19:00, 19:05, 19:07 and were terminated by normal polarity lightning flashes, see Fig. 1a. TGE started at 19:08 characterized by a long duration ( $\approx 20$ minutes) with electric field reversals, see Fig. 1a. We suppose that the TGE start at 19:08-19:11 was controlled by the mature LPCR that produced a positive near-surface electric field. LPCR is a transient structure located at the bottom of the cloud. The height of the cloud estimated from the outside temperature and the duw point was $150 \mathrm{~m}$.

The LPCR and main negatively charged layer, positioned usually $2-3 \mathrm{~km}$ higher in the middle of the cloud formed dipole which accelerated electrons downward in the direction of the earth. As the bottom of the cloud was rather high above the earth's surface where particle detectors were located, the intensity of TGE was rising rather slowly. After the near-surface field polarity reversal from positive to negative at 19:11, the atmospheric electric field was controlled by the main negative charge region only, and during 7 minutes the field strength remained below $-20 \mathrm{kV} / \mathrm{m}$. During this time interval, the particle flux continued to rise above the background reaching 8.5 percent enhancement above the background. At 19:18 another near-surface electric field polarity reversal from negative to positive occurred and the near-surface electric field was controlled again by a newly formed LPCR. As a result, the field strength remained $\approx 15 \mathrm{kV} / \mathrm{m}$ for 10 minutes. All this time particle flux slowly decayed, staying constant for a few minutes, and finally terminated at 19:31. Another possible scenario of atmospheric electric field dynamic is connected with the end of storm oscillations (EOSO). At the end of the storm charged layers are consequently grounded and at several moments the main positive charge region and its negatively charged screening layer, that at the main phase of the storm is located high at the top of the cloud, go down and form an "inverse" dipole and continue to accelerate seed electrons downward. 
The structure of the electric field of the storm that occurred on 25 September was much simpler, see Fig. 1b. A large negatively charged region in the middle of the cloud controls the atmospheric electric field and provides a much larger TGE than the first event discussed above ( $20 \%$ enhancement above background) lasting $\approx 5$ minutes until normal polarity lightning flash abruptly terminates it (distance to lightning flash was $\approx 5 \mathrm{~km}$ ). The near-surface electric field was in a deep negative domain (below $-20 \mathrm{kV} / \mathrm{m}$ ) during TGE. Although the cloud base was located rather high at $400 \mathrm{~m}$., there were no signs that an LPCR is formed. Usually, outbursts of the near-surface electric field reaching the positive domain occurred during the deep negative field period when the LPCR formed. Surely, LPCR significantly increased the electric field within the cloud, but it decreases, and sometimes reverses the electric field between LPCR and earth's surface; thus, the RREA terminates. If the cloud is high above detectors, for instance, $400 \mathrm{~m}$, we cannot expect that electrons will reach the earth's surface and registered. However, we observe electron flux at 19:42. Thus, there was no LPCR formed, and the strong electric field extended deeper in the atmosphere below the cloud.

In Fig. $2 \mathrm{a}$ and $\mathrm{b}$ we present differential energy spectra of these two TGE events for the minute of the highest flux. Three $20 \mathrm{sec}$. histograms were joint to form a 1-minute histogram of the energy releases in a $60 \mathrm{~cm}$ thick scintillator for the further recovering of the energy spectra using the response function of spectrometer obtained with GEANT 4 simulation.

To check recovered energy spectra, we compare expected count rates with count rates measured at the same minute by other particle detectors located at distance of 20 and $100 \mathrm{~m}$ from the ASNT detector. For comparison, we use two other particle detectors located at Aragats, namely $5 \mathrm{~cm}$ thick and $1 \mathrm{~m}^{2}$ area plastic scintillator with energy threshold $\approx 4 \mathrm{MeV}$; and $3 \mathrm{~cm}$ thick scintillator with the same area and with energy threshold $\approx 2 \mathrm{MeV}$. In Fig. $3 \mathrm{a}$ and $\mathrm{b}$ we show the simple calculation procedure of the expected count rate of both scintillators.

In Fig 3a we show the procedure of estimation of the expected flux of electrons and gamma rays with energies above $2 \mathrm{MeV}$; and in Fig $3 \mathrm{~b}$ - with energies above $4 \mathrm{MeV}$. In Table 1 we show values of particle fluxes with 2 energy thresholds and corresponding count rates measured by 3 and $5 \mathrm{~cm}$ thick scintillators.

Table 1. Estimated and measured count rates of 2 plastic scintillators at minute of maximum TGE flux

\begin{tabular}{|c|c|c|c|c|c|c|c|c|}
\hline \multirow[t]{3}{*}{ Date } & \multirow{2}{*}{\multicolumn{2}{|c|}{$\begin{array}{l}\text { Integral } \\
\text { Spectrum } \\
E>=2 \mathrm{MeV}\end{array}$}} & \multirow{2}{*}{\multicolumn{2}{|c|}{$\begin{array}{l}\text { Integral } \\
\text { Spectrum } \\
\text { E>=4MeV }\end{array}$}} & \multirow{3}{*}{$\begin{array}{l}\text { Expected } \\
\text { count } \\
\text { rate }>2 \\
\text { MeV }\end{array}$} & \multirow{3}{*}{$\begin{array}{l}\text { Count } \\
\text { rate } \\
\text { STAND3 } \\
\text { upper }\end{array}$} & \multirow{3}{*}{$\begin{array}{l}\text { Expected } \\
\text { count } \\
\text { rate >4 } \\
\mathrm{MeV}\end{array}$} & \multirow{3}{*}{$\begin{array}{l}\text { Count } \\
\text { rate } \\
\text { SEVAN } \\
\text { upper }\end{array}$} \\
\hline & & & & & & & & \\
\hline & Elect. & $\begin{array}{l}\text { Gamma } \\
\text { rays }\end{array}$ & Elect. & $\begin{array}{l}\text { Gamma } \\
\text { rays }\end{array}$ & & & & \\
\hline 09.25 .2020 & 6800 & 96200 & 3980 & 26000 & 10650 & 11800 & 5440 & 5400 \\
\hline 06.27 .2020 & 1550 & 45000 & 1110 & 15000 & 3350 & 3300 & 2010 & 2000 \\
\hline
\end{tabular}


We estimated the expected count rate by multiplying the incident particle flux by the detection efficiency of electrons and gamma rays. We assume $99 \%$ efficiency for electron registration for both $3 \mathrm{~cm}$ thick and $5 \mathrm{~cm}$ thick scintillators and $4 \%$ and $6 \%$ efficiency to register gamma rays for 3 and $5 \mathrm{~cm}$ scintillator, respectively. Very good agreement of calculated and measured count rates also indicates that the RREA particle flux is rather stable on distances of $\approx 100 \mathrm{~m}$.

\section{Corsika Simulations Of The Rrea Process Above Aragats Station}

To understand the avalanche development in the electrified atmosphere and to compare measured energy spectra with simulated ones we used the CORSIKA version 7.7400, which takes into account the effect of the electric field on the transport of particles. As it was already demonstrated by CORSIKA and GEANT4 simulations [18], the multiplication and acceleration of electrons of cosmic rays, namely the RREA process [3] is a threshold process and avalanches can be started when the atmospheric electric field exceeds the threshold value that depends on the air density (for instance $\approx 1.7 \mathrm{kV} / \mathrm{cm}$ at $5000 \mathrm{~m}$ ). The extent of the electric field also should be sufficiently large to ensure avalanche development. The simulation of the RREA was done within the vertical region of the uniform electric field with strengths exceeding the runaway breakdown threshold by a few tens of percent on 1-3 km height above Aragats station. The assumed uniformity of the electric field extending up to $3 \mathrm{~km}$ leads to the change of the surplus to threshold energies at different heights according to particular air density value. Thus, the $2.2 \mathrm{kV}$ at $4.9 \mathrm{~km}$ height is $\approx 20 \%$ larger than critical energy, and at $3.4 \mathrm{~km}$ height is only $\approx 10 \%$ larger. Thus, the critical energy smoothly decreases with the increase of the depth in the atmosphere. We do not test electrical fields stronger than $2.2 \mathrm{kV} / \mathrm{m}$. The development of RREA will certainly increase the electrical conductivity in the cloud. In numerous studies (Marshal et al., 2005, Gunn, 1947) was shown that lightning flash occurs after the RREA initialization threshold exceeds $10-20 \%$. The RREA simulation codes do not include the lightning initialization mechanism, thus one can exceed the strength of the electric field above any reliable value to get billions and billions of avalanche particles, but it is not physically justified.

The largest TGEs occurred when the distance to the cloud base was $25-100 \mathrm{~m}$ (see Fig 17 in [10]), thus in our simulations, the electromagnetic avalanche continued propagation in the dense air additionally 25 , 50,100 , and 200 meters before registration. To avoid abrupt nonphysical termination of the electric field we keep the field strength below the critical value (say $1 \mathrm{kV} / \mathrm{cm}$ ) till particles reached detectors. To avoid contamination by the MOS process (modification of electron energy spectra, see details in [19]) simulations for simplicity were performed with vertical beams of $1 \mathrm{MeV}$ electrons, as seed particles for the relativistic runaway avalanche. In this way we obtain estimates of the maximum energy achieved by the RREA gamma rays; the MOS process generates additional high-energy bremsstrahlung gamma rays from high energy electrons of the ambient population of cosmic rays. The electron energy spectrum at energies corresponding to the minimum of ionization losses is rather flat, thus our approximation does not introduce large bias. Simulation trials include from $10^{3}$ to $10^{4}$ events for the electric field strengths of $1.9-2.2 \mathrm{kV} / \mathrm{cm}$. The propagation of electrons and gamma rays were followed in the avalanche until their 
energy decreased down to $0.05 \mathrm{MeV}$. The energy spectra of RREA electrons and gamma rays were obtained as a result of each simulation trial. In simulation trials, we follow the development of the RREA where we show the number of electrons and gamma rays in RREA per seed electron on different stages of avalanche development in the strong electric field and after leaving the strong electric field region, see Fig.4 a) and b). From the figures, we can see that for large electric field strengths the number of electrons exceeds the number of gamma rays, however, after the exit from the electric field electron flux rapidly attenuates (see Fig. 4a) and, at $100 \mathrm{~m}$ below the strong electric field, the number of gamma rays exceeds the number of electrons.

In Table 2 we show the parameter of 2 TGEs registered in 2020 and similar parameters of CORSIKA simulations of the RREA process in the atmosphere. In the second column, we show the number of electrons and gamma rays in RREA simulation per seed electron on different distances from the exit of the strong electric field and the number of electrons and gamma rays registered in the experiment. In the third column, we show the height of cloud assumed in simulation and estimated in the experiment. In the fourth column, we show the number electron to gamma ray ratio for particles with energies above $7 \mathrm{MeV}$ for simulation and experiment.

In the fifth and sixth columns, we put the index of the power-law fit and maximal energy of the electron energy spectrum, respectively. The seventh and eighth columns show the same parameters for the gamma ray energy spectrum.

Table 2. Parameters of the RREAs calculated with CORSIKA code and of 2 TGES observed in 2020.

\begin{tabular}{|c|c|c|c|c|c|c|c|c|}
\hline & $\begin{array}{l}\mathrm{N} \text { of el. } \\
\text { E>7 } \\
\mathrm{MeV} \\
\text { per } \\
\text { seed } \\
\text { electron }\end{array}$ & $\begin{array}{l}\mathrm{N} \text { of } \mathrm{g} \\
\text { rays E>> } \\
7 \mathrm{MeV} \\
\text { per } \\
\text { seed } \\
\text { electron }\end{array}$ & $\begin{array}{l}\text { Height } \\
\text { of the } \\
\text { cloud } \\
\text { base }\end{array}$ & $\begin{array}{l}\mathrm{Ne} / \mathrm{Ng} \\
>7 \\
\mathrm{MeV}\end{array}$ & $\begin{array}{l}\text { Power } \\
\text { law } \\
\text { index: } \\
\text { electrons } \\
>7 \mathrm{MeV}\end{array}$ & $\begin{array}{l}\text { Max. } \\
\text { energy } \\
\text { electrons } \\
\text { MeV }\end{array}$ & $\begin{array}{l}\text { Power } \\
\text { law } \\
\text { index } \\
\text { gamma } \\
\text { ray }>7 \\
\text { MeV }\end{array}$ & $\begin{array}{l}\text { Max. } \\
\text { Energy } \\
\text { Gamma } \\
\text { rays } \\
\text { MeV }\end{array}$ \\
\hline $\begin{array}{l}2.0 \mathrm{kV} / \mathrm{cm}, 2 \\
\mathrm{~km}\end{array}$ & 0.57 & 1.03 & 50 & 0.55 & -3.83 & 31 & -3.82 & 31 \\
\hline $\begin{array}{l}2.0 \mathrm{kV} / \mathrm{cm}, 2 \\
\mathrm{~km}\end{array}$ & 0.04 & 0.87 & 100 & 0.046 & -3.75 & 20 & -3.73 & 31 \\
\hline $\begin{array}{l}2.0 \mathrm{kV} / \mathrm{cm}, 2 \\
\mathrm{~km}\end{array}$ & 0.013 & 0.77 & 200 & 0.017 & -4.45 & 17 & -3.61 & 29 \\
\hline 09.25 .2020 & 0.087 & 0.3 & 400 & 0.28 & -1.48 & 30 & -2.6 & 40 \\
\hline 06.27 .2020 & 0.028 & 0.2 & 100 & 0.014 & -1.8 & 30 & -2.9 & 35 \\
\hline
\end{tabular}


As we can see in the Fig. 4 and in Table 2, the energy spectra of the large TGE registered on 25 September 2020 is well matching Monte Carlo calculations of the RREA developing in the $2 \mathrm{~km}$ extended electric field of $2.0 \mathrm{kV} / \mathrm{cm}$ strength, which ends $\approx 100 \mathrm{~m}$ above earth's surface.

\section{Discussion And Conclusions}

We measure energy spectra of TGE electrons and gamma rays with large scintillation spectrometers, estimate parameters of the power-law fit and cloud height above detectors.

We confirm the obtained spectra by calculated expected count rates of other particle detectors independently measuring particle fluxes on Aragats and obtain rather good agreement (see Table 1). Afterward, we perform a cycle of simulations of the RREA process in the electric fields exceeding the runaway threshold by $10-20 \%$ and extended $1-3 \mathrm{~km}$ above particle detectors.

The results of the comparisons are shown in Fig. 4 and Table 2. We select from the simulations ones thus parameters which are most close to the TGE observed on 25 September 2020 ( $2 \mathrm{~km}$ field extension and 2 $\mathrm{kV} / \mathrm{cm}$ uniform electric field). However, we can choose other combinations of these parameters which are also compatible with observations.

The number of RREA electrons (per one seed electron) abruptly decreases as avalanche exits from the region of the strong electric field (from 0.57 at $50 \mathrm{~m}$ below field to 0.013 at $200 \mathrm{~m}$ ). The number of electrons ( 0.087 and 0.028$)$ measured in TGE fits within simulated values. The simulated number of gamma rays for distances 50-100 m exceeds the measured values more than 2 times. The ratio of electron to gamma rays for RREA and TGE coincides around $100 \mathrm{~m}$ for both observed TGEs. Power law indices differ from each other significantly, especially for electrons. The very sharp decrease of electrons after the exit from the electric field confirmed in the experiment by the low electron-to-gamma ray ratio, is not consistent with very hard recovered energy spectra. In contrast, the maximum energies of the simulated and observed spectra are in good agreement, and the relation between them (maximum energies of gamma rays exceed maximum energies of electrons) obtained in simulations is confirmed by the observations as well. Observed maximum energies also indicate that a strong electric field is terminated around $100 \mathrm{~m}$ above the earth's surface.

We can conclude that the variants of field topology and strengths assumed in simulations are too simplified to reproduce the rather complicated and dynamic nature of the atmospheric electric field. Nonetheless, the closeness of several measured and observed parameters allows us to confirm that the RREA is the origin of the TGE and to outline the most probable characteristics of the atmospheric electric field for particular observed TGE events.

\section{Declarations}


We thank the staff of the Aragats Space Environmental Center for the operation of the ASNT detector on Mount Aragats. A. C. thanks S. Soghomonyan for the valuable comments and for the useful, multiyear discussions on the origin of the atmospheric electric field. The authors thank $\mathrm{S}$. Chilingaryan for his continuous efforts at maintaining and improving Web-based data analysis facilities for a large stream of data coming online from the Mount Aragats research station.

\section{References}

1. Alexeenko VV, Khaerdinov NS, Lidvansky AS et al (2002) Transient variations of secondary cosmic rays due to atmospheric electric field and evidence for pre-lightning particle acceleration. Phys Lett $A$ 301:299-306

2. Babich LP (2017a) Radiocarbon production by thunderstorms. Geophys Res Lett 44:11191-11200

3. Babich LP (2017b) Thunderous nuclear reactions. Nature 551:443-444 Babich LP, Donskoy EN, Kutsyk IM et al (2001) Comparison of relativistic runaway electron avalanche rates obtained from monte carlo simulations and from kinetic equation solution. IEEE Trans Plasma Sci 29:430-438

4. Babich LP, Donskoy EN, Il'kaev RI, Kutsyk IM, Roussel-Dupre' RA (2004) Fundamental parameters of a relativistic runaway electron avalanche in air. Plasma Phys Rep 30:616-624

5. Briggs MS, Connaughton V, Wilson-Hodge $C$ et al (2011) Electron- positron beams from terrestrial lightning observed with Fermi GBM. Geophys Res Lett 38:L02808

6. Chilingarian A, Daryan A, Arakelyan K et al (2010) Ground-based observations of thunderstormcorrelated fluxes of high-energy electrons, gamma rays, and neutrons. Phys Rev D 82:043009 Chilingarian A, Hovsepyan G, Hovhannisyan A (2011) Particle bursts from thunderclouds: natural particle accelerators above our heads. Phys Rev D Part Fields 83(6):062001

7. Chilingarian A, Bostanjyan N, Vanyan L (2012) Neutron bursts associated with thunderstorms. Phys Rev D Part Fields 85(8):085017

8. Chilingarian A, Hovsepyan G, Kozliner L (2013a) Thunderstorm ground enhancements: gamma ray differential energy spectra. Phys Rev D Part Fields 88:073001هChilingarian A, Bostanjyan N, Karapetyan T (2013b) On the possibility of location of radiation-emitting region in thundercloud. J Phys Conf Ser 409:012217

9. Chilingarian A, Hovsepyan G, Mantasakanyan E (2016) Mount Aragats as a stable electron accelerator for atmospheric high-energy physics research. Phys Rev D Part Fields 93(5):052006

10. Chilingarian A, Khanikyants $Y$, Mareev E et al (2017a) Types of lightning discharges that abruptly terminate enhanced fluxes of energetic radiation and particles observed at ground level. J Geophys Res Atmos 122:7582-7599

11. Chilingarian A, Chilingaryan $S$, Karapetyan $T$ et al (2017b) On the initiation of lightning in thunderclouds. Sci Rep 7(1):1371. https ://doi.org/10.1038/s41598-017-01288-0(2017).

12. Chilingarian A, Hovsepyan G, Mailyan B (2017c) In situ measurements of the runaway breakdown (RB) on Aragats mountain. Nucl Inst Methods Phys Res A 874:19-27. 
13. Chum et al., Significant enhancements of secondary cosmic rays and electric field at the high mountain peak of Lomnický Štít in High Tatras during thunderstorms, Earth, Planets and Space (2020) 72:28 https://doi.org/10.1186/s40623-020-01155-9

14. Dwyer JR (2003) A fundamental limit on electric fields in air. Geophys Res Lett 30(20):2055.

15. Eack KB, Beasley WH, Rust WD et al (1996) Initial results from simul- taneous observations of $X$ rays and electric fields in a thunder- storm. J Geophys Res 101:29637-29640

16. Enoto T, Wada $Y$, Furuta $Y$ et al (2017) Photonuclear reactions trig- gered by lightning discharge. Nature (London) 551:481.

17. Fishman GJ, Bhat PN, Mallozzi V et al (1994) Discovery of intense gamma ray flashes of atmospheric origin. Science 264:1313 Gurevich AV, Milikh GM, Roussel-Dupre R (1992) Runaway electron mechanism of air breakdown and preconditioning during a thunderstorm. Phys Lett $A$ 165:463.

18. Gunn, R. (1947) Electric field intensity inside of natural clouds. J. Appl. Phys., 19, 481-484.

19. A.V. Gurevich, K.P. Zybin, R.A. Roussel-Dupre, Lightning initiation by simultaneous of runaway breakdown and cosmic ray showers, Phys. Lett. A 254 (1999) 79.

20. Gurevich AV et al (2012) Strong flux of low-energy neutrons produced by thunderstorms. Phys Rev Lett 108:125001

21. Kelley NA, Smith DM, Dwyer JR et al (2015) Relativistic electron avalanches as a thunderstorm discharge competing with lightning. Nature Commun 6:7845.

22. Kochkin $P$, van Deursen PJ, Marisaldi $M$ et al (2017) In-flight observation of gamma ray glows by ILDAS. JGR, Atmos. 122:12801-12811.

23. Kudela K, Chum J, Kollárik M, Langer R, Strhárský I, Baše J (2017) Correlations between secondary cosmic ray rates and strong electric fields at Lomnický štít. J Geophys Res Atmos 122(10):7001070710

24. Kuroda Y, Oguri S, Kato $Y$ et al (2016) Observation of gamma ray bursts at ground level under the thunderclouds. Phys Lett B 758:286-291

25. Mailyan BG, Briggs MS, Cramer ES et al (2016) The spectroscopy of individual terrestrial gamma-ray flashes: constraining the source properties. JGR Sapce Phys 121:11346-11363.

26. McCarthy MP, Parks GK (1985) Further observations of X-rays inside thunderstorms. Geophys

27. A.Shepetov, V.Antonova, O. Kalikulov, et al. (2021) The prolonged gamma ray enhancement and the short radiation burst events observed in thunderstorms at Tien Shan, Atmospheric Research, 248, 105266.

28. Res Lett 97:5857-5864. https://doi. org/10.1029/GL012i006p00393.

29. Tavani $\mathrm{M}$ et al (2011) (AGILE team), terrestrial gamma-ray flashes as powerful particle accelerators. Phys Rev Lett 106:018501

30. Torii T, Sugita T, Kamogawa M et al (2011) Migrating source of ener- getic radiation generated by thunderstorm activity. Geophys Res Lett 38:L24801 
31. Tsuchiya H, Hibino K, Kawata K et al (2012) Observation of thun- dercloud-related gamma rays and neutrons in Tibet. Phys Rev D 85:092006

32. Tsuchiya $\mathrm{H}$, Enoto $\mathrm{T}$, Iwata $\mathrm{K}$ et al (2013) Hardening and termination of long-duration g rays detected prior to lightning. Phys Rev Lett 111:015001

33. Shi, D., Wang, D., Wu, T., \& Takagi, N. (2019). Correlation between the first return stroke of negative CG lightning and its preceding discharge processes. Journal of Geophysical Research: Atmospheres, 124. https://doi.org/ 10.1029/2019JD030593

\section{Figures}

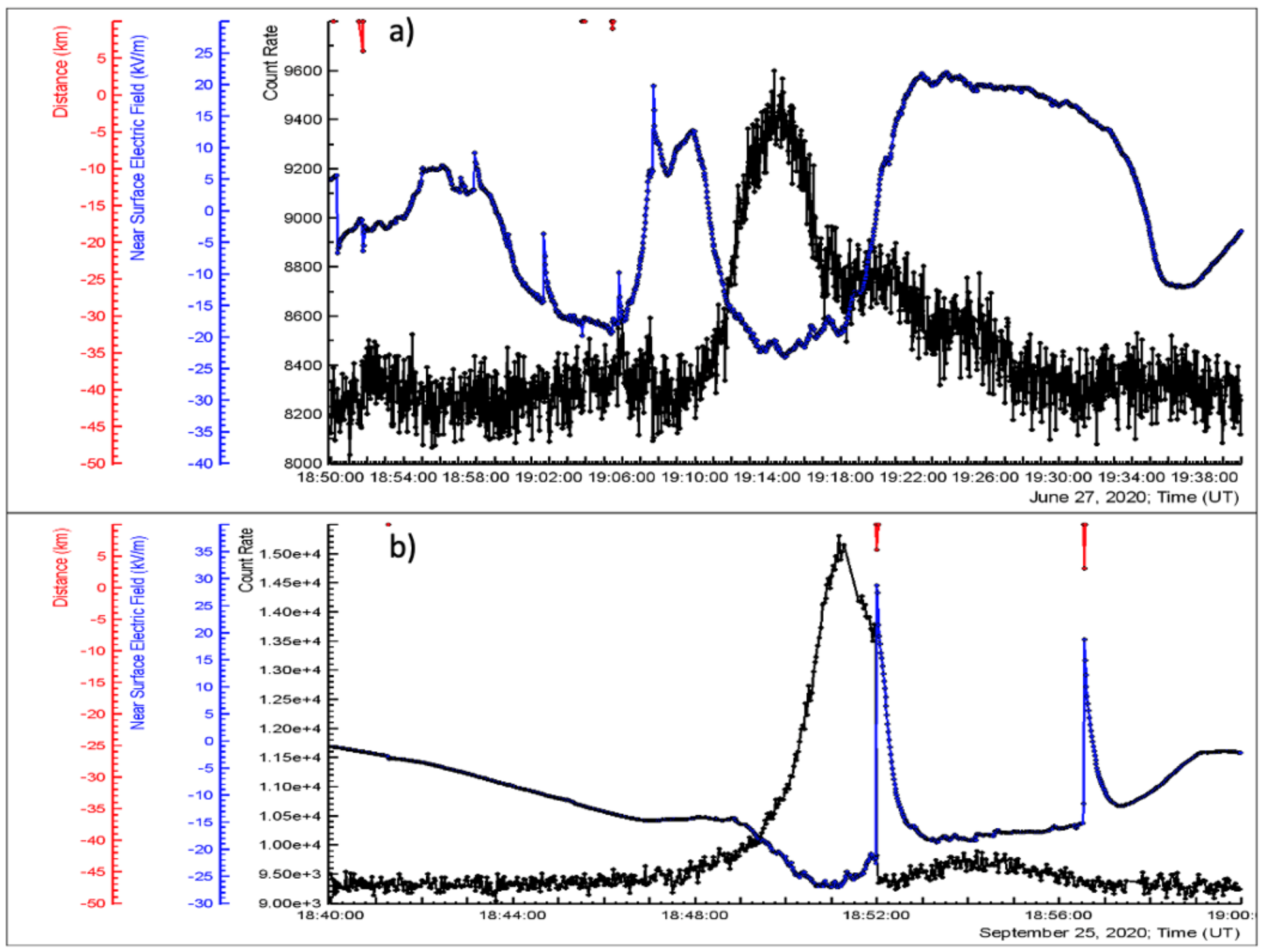

Figure 1

The time series of count rate measured by a $60-\mathrm{cm}$ thick scintillator (black) and disturbances of the nearsurface electric field (blue). In the top of frames by red lines, the distance to lightning flash is shown. 


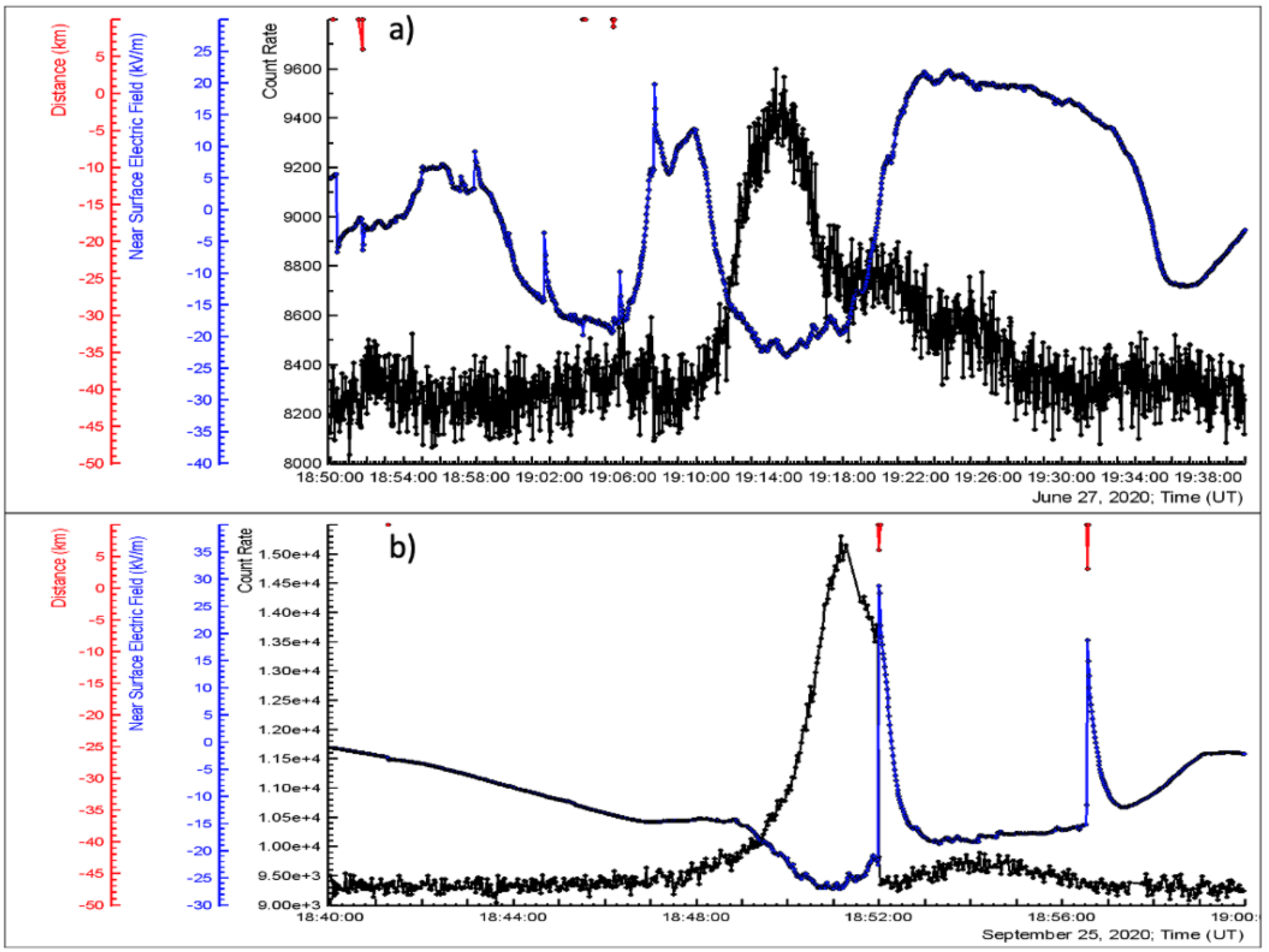

Figure 1

The time series of count rate measured by a $60-\mathrm{cm}$ thick scintillator (black) and disturbances of the nearsurface electric field (blue). In the top of frames by red lines, the distance to lightning flash is shown. 

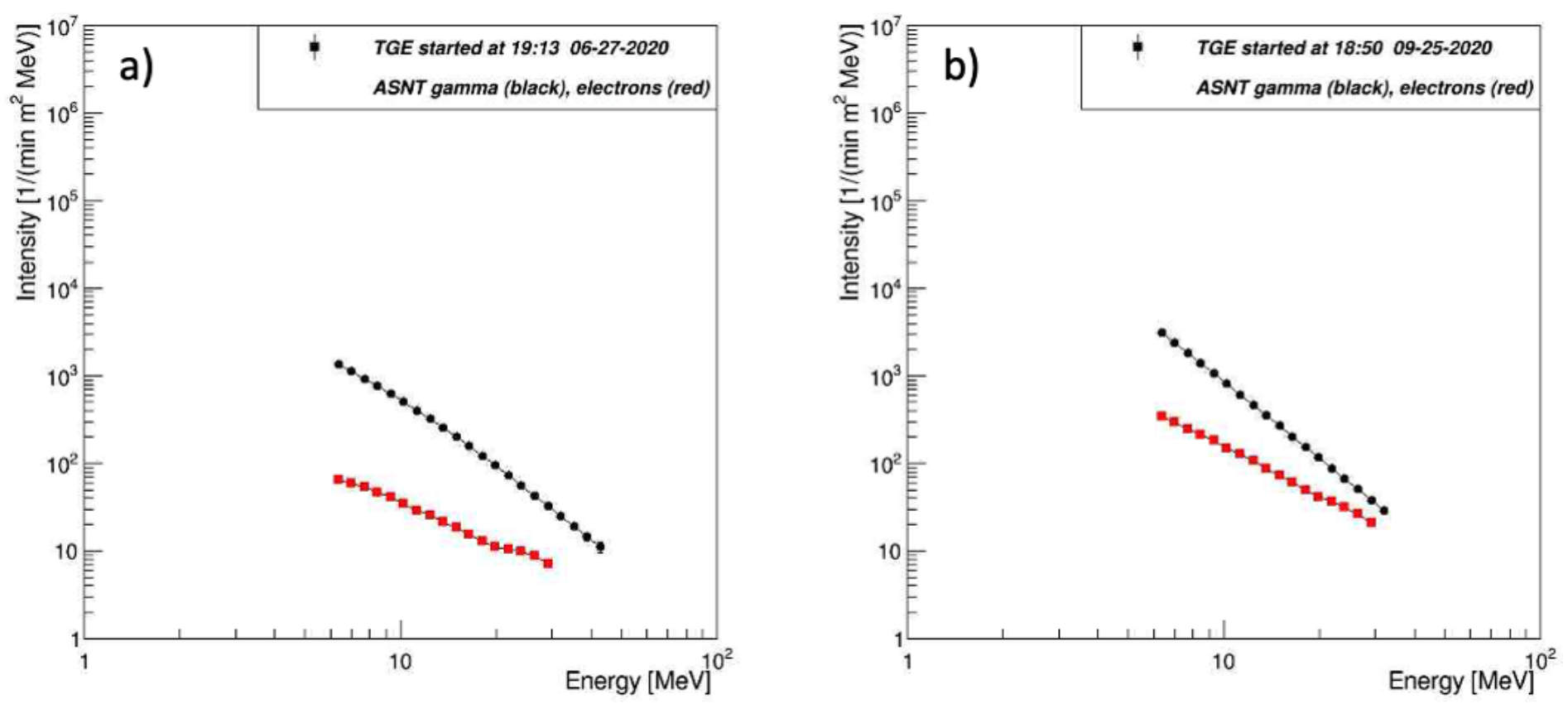

\section{Figure 2}

Differential energy spectra of RREA electrons (red) and gamma rays (black) measured by ASNT spectrometer at altitude $3200 \mathrm{~m}$ at highest particle flux above $7 \mathrm{MeV}$.
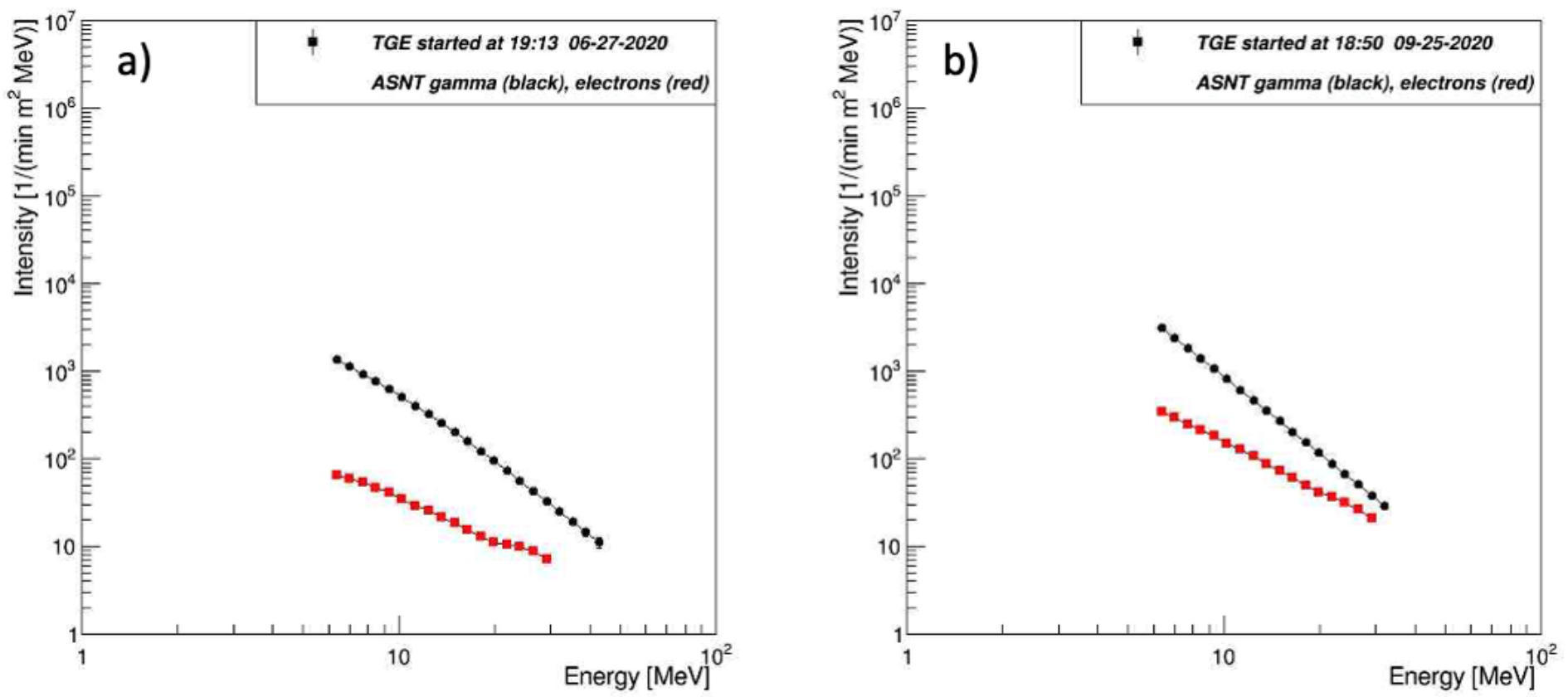

\section{Figure 2}

Differential energy spectra of RREA electrons (red) and gamma rays (black) measured by ASNT spectrometer at altitude $3200 \mathrm{~m}$ at highest particle flux above $7 \mathrm{MeV}$. 

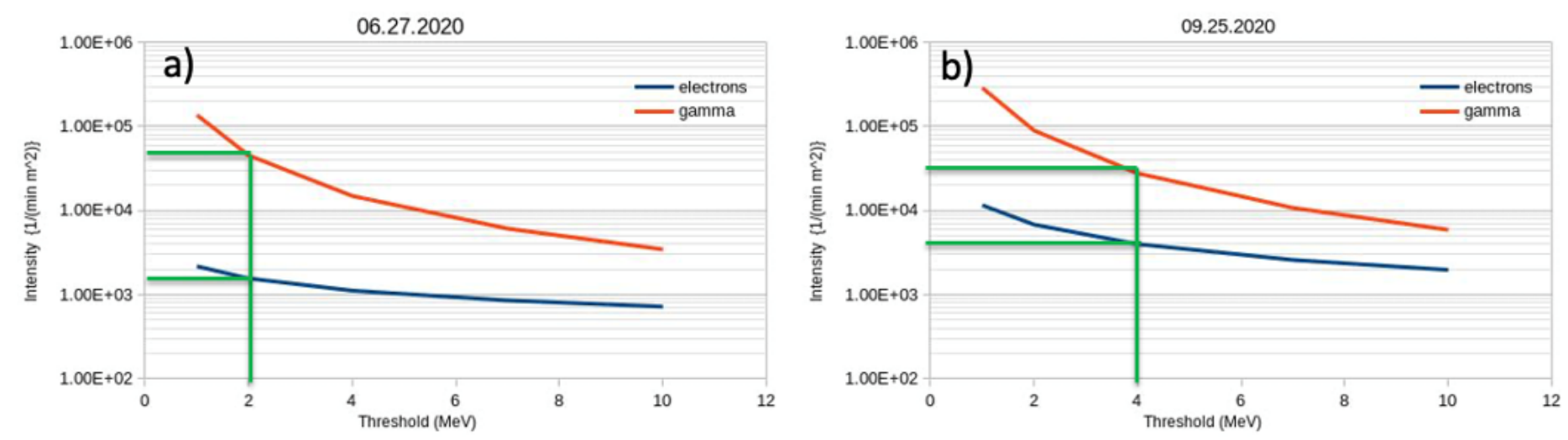

\section{Figure 3}

Integral energy spectra of 2 TGE events. By vertical and horizontal green lines, we explain the calculation of expected count rates, which is directly readout from the vertical axis of figures.
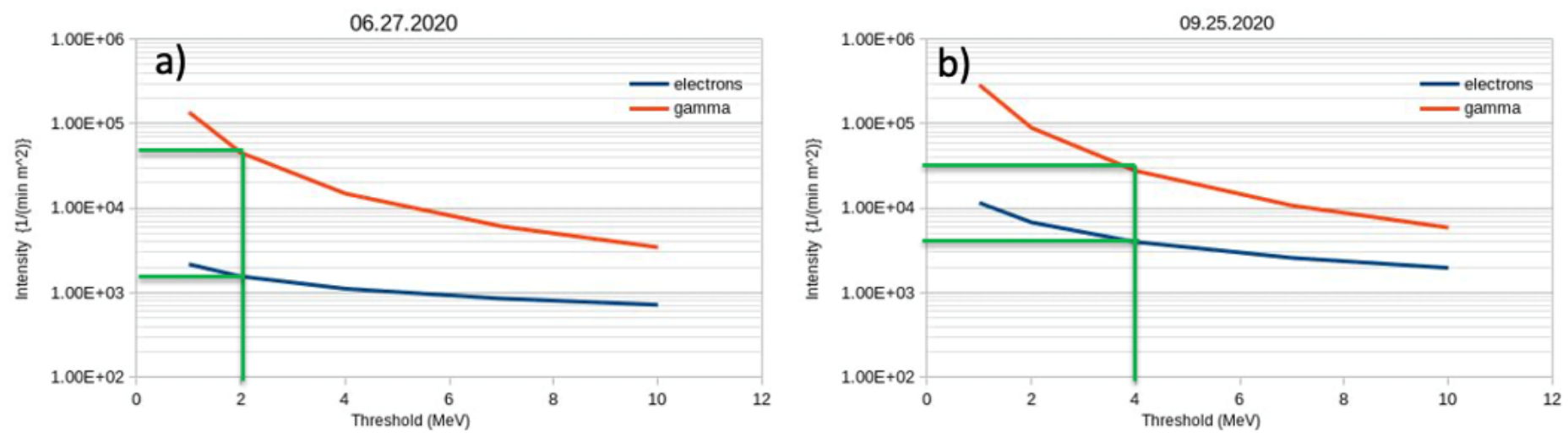

Figure 3

Integral energy spectra of 2 TGE events. By vertical and horizontal green lines, we explain the calculation of expected count rates, which is directly readout from the vertical axis of figures.
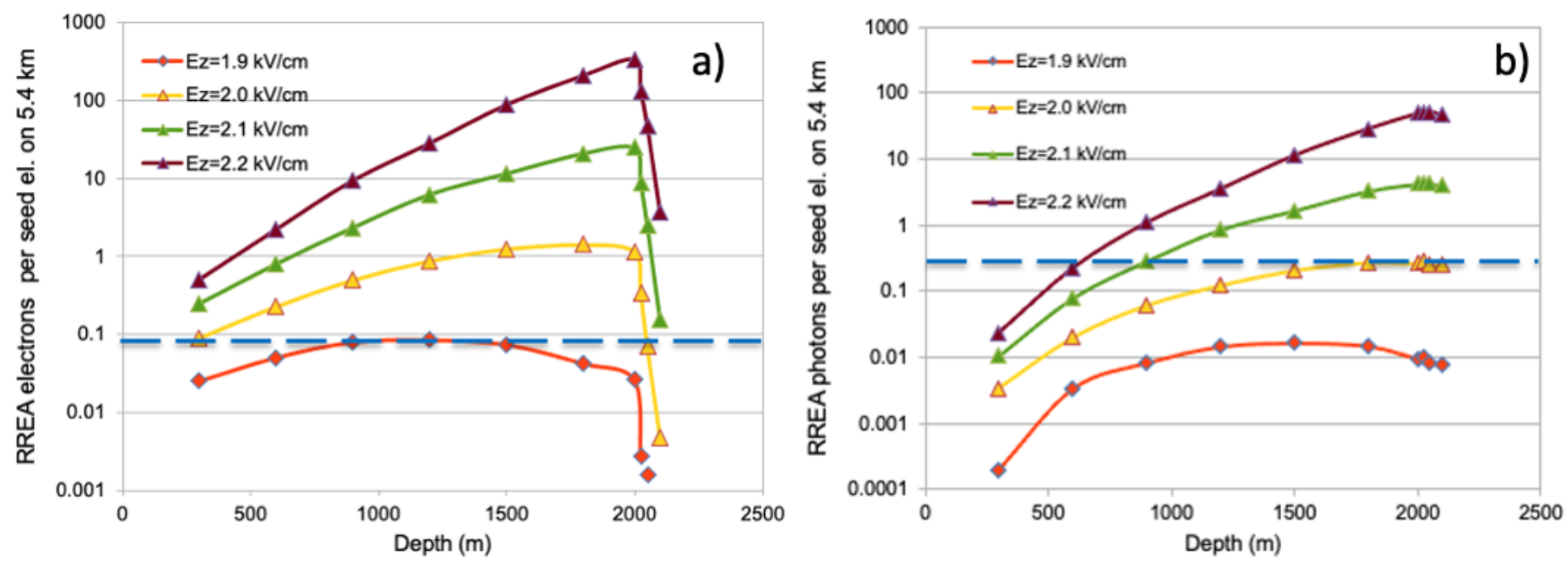

Figure 4 
Development of the electromagnetic avalanche in the atmosphere. Avalanche started at $5400 \mathrm{~m}, 2.2 \mathrm{~km}$ above the Aragats station. The number of avalanche particles is calculated each $300 \mathrm{~m}$. After exiting from the electric field propagation of avalanche particles are followed additionally $200 \mathrm{~m}$ before reaching the station. By blue dashed line, we show the electron and gamma ray number per seed electron for the TGE that occurred on 25 September 2020.
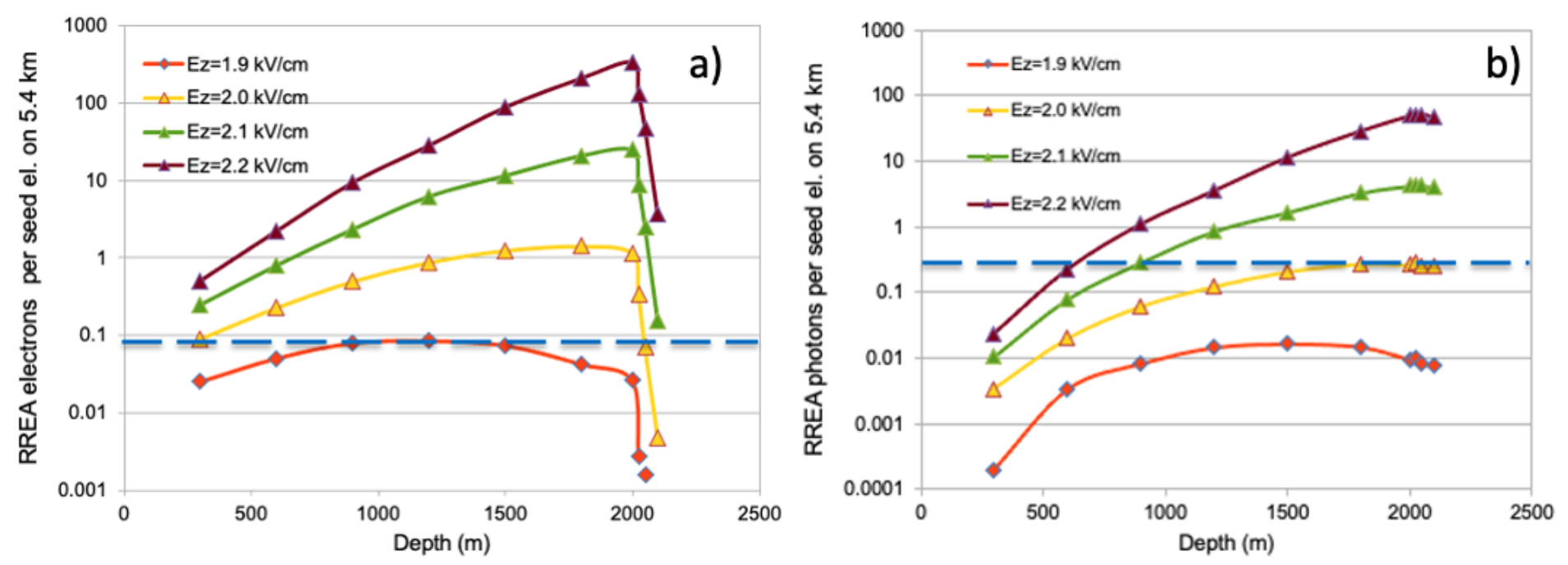

\section{Figure 4}

Development of the electromagnetic avalanche in the atmosphere. Avalanche started at $5400 \mathrm{~m}, 2.2 \mathrm{~km}$ above the Aragats station. The number of avalanche particles is calculated each $300 \mathrm{~m}$. After exiting from the electric field propagation of avalanche particles are followed additionally $200 \mathrm{~m}$ before reaching the station. By blue dashed line, we show the electron and gamma ray number per seed electron for the TGE that occurred on 25 September 2020. 